\title{
Wechselnde Mehrheiten im Landtag von Nordrhein-Westfalen. Wie werden demokratisch legitimierte Mehrheitsverhältnisse bei parlamentarischen Abstimmungen gesichert?
}

\section{André Vielstädte}

Parlamentarische Mehrheiten entstehen aus demokratischen Wahlen, die Mandatsverteilung resultiert aus nackten Zahlen, der Summe der Stimmzettel. Prima facie dürfte man folglich annehmen, dass die Feststellung von Abstimmungsergebnissen im Parlament eine reine Formsache ist. Bei näherem Hinsehen beeinflussen aber - neben beispielsweise Fraktionsdisziplin, Krankheitsausfällen oder der Unabhängigkeit der Abgeordneten - weitere Faktoren den Ausgang von parlamentarischen Abstimmungen. Im Folgenden soll es vor allem darum gehen, dass der Parlamentspräsident mit seinem Sitzungsvorstand wie auch die Methode der Abstimmung das zahlenmäßige Ergebnis beeinflussen können.

Die Ausgangslage für diese Hypothese bietet der Landtag von Nordrhein-Westfalen in seiner aktuellen Legislaturperiode seit Juni 2010. Mit wechselnden parlamentarischen Mehrheiten gelingt es der Minderheitsregierung von SPD und Bündnis 90/Die Grünen bislang, parlamentarische Beschlüsse zu fassen und somit handlungsfähig zu sein. Dass wechselnde Mehrheiten nicht nur bei der Zusammenarbeit zwischen Oppositionsfraktionen und der Regierungskoalition, sondern auch innerhalb eines Abstimmungsprozesses zustande kommen können, zeigt der folgende Fall.

\section{Die Ausgangssituation}

Am 19. Mai 2011 nahm die nordrhein-westfälische Minderheitsregierung die wichtige Hürde des ersten eigenen Haushaltes. In dritter Lesung verabschiedete der Landtag den Haushalt 2011 mit einfacher Mehrheit von SPD und Bündnis 90/Die Grünen. Die Enthaltung der Fraktion Die Linke genügte, trotz Gegenvotum von CDU und FDP, zur Gesetzesverabschiedung. Die medial hochstilisierte Abstimmung im Düsseldorfer Landtag dauerte nur wenige Augenblicke, obwohl daran - so der Medientenor - die Existenz der rot-grünen Minderheitsregierung hing. Der Aufruf des Tagesordnungspunktes durch die sitzungsleitende Landtagsvizepräsidentin Gunthild Böth (Linke) und die darauf folgende Frage an das Plenum, wer zustimmt, sich enthält oder ablehnt, war reines Protokoll. Die Mandatsträger hoben den Arm zur Abstimmung, und abschließend verkündete Böth das Ergebnis.

Am folgenden Sitzungstag führte das Verhalten der noch am Vortag souveränen und protokollarisch präzise handelnden Landtagsvizepräsidentin zu einem Eklat im Düsseldorfer Plenarsaal. Am Nachmittag stand die Abstimmung eines Antrags der FDP-Fraktion zum Thema „Kraftwerk Datteln IV jetzt zügig fertigstellen“ auf der Tagesordnung. ${ }^{1}$ Das Thema bot politischen Sprengstoff, da die Koalitionspartner beim Kohlekraftwerksbau in Datteln unterschiedliche Meinungen vertraten. Dennoch hatten sie im Vorfeld die Absprache getroffen, den Antrag abzulehnen; dem wollte sich auch die Linke anschließen. Damit lag ein typischer Antrag von Oppositionsfraktionen im Parlament vor, der nur selten

1 Vgl. Landtag Nordrhein-Westfalen, Plenarprotokoll 15/35. 
öffentlich wahrgenommen wird. Letzter Redner vor Abstimmungsbeginn am Nachmittag war der Fraktionsvorsitzende der FDP Gerhard Papke. Laut Rednerliste sprach anschließend noch Rüdiger Sagel von der Linken, der jedoch kurzfristig auf seine Rede verzichtete. Dabei ist entscheidend zu bemerken, dass der Plenarsaal zu diesem Zeitpunkt lediglich von den Fachpolitikern besetzt war. Im parlamentarischen Alltag ist es üblich, dass die Abgeordneten rund zehn Minuten vor Beginn einer Abstimmung per Kurznachricht informiert werden. Daraufhin füllt sich der Plenarsaal und die parlamentarischen Mehrheiten verfestigen sich. Aufgrund des Redeverzichtes kam es in dieser Situation jedoch zur verfrühten Abstimmung.

Die Vizepräsidentin bat um die Stimmen, woraufhin die Abgeordneten ihre Hände zum Votum hoben. Sie stellte fest, dass CDU und FDP für den Antrag stimmten, SPD, Grüne sowie Linke dagegen. Dieses Ergebnis hätte bei Vollständigkeit der Fraktionen einer Ablehnung des Antrages bei $80 \mathrm{zu} 101$ Stimmen entsprochen. ${ }^{2} \mathrm{Zu}$ einer Feststellung des Ergebnisses kam es jedoch nicht. Das Wortprotokoll dokumentiert die Ausrufe der Opposition, eine Mehrheit zu besitzen. Böth schaute in diesem Moment nickend Richtung FDP und CDU und sagte: „Jetzt haben wir ein Problem, wenn wir die Türen nicht schließen.“ Fernsehbilder dokumentieren im Nachhinein eine eindeutige Mehrheit von CDU und FDP im Plenarsaal. ${ }^{3}$ Im weiteren Verlauf bat Böth indirekt, die Türen des Plenarsaals zu schließen: „Es geht in einem solchen Fall überhaupt nicht - da stimme ich Ihnen völlig zu - dass Abgeordnete in den Plenarsaal kommen, nachdem wir diese Abstimmung hatten. [...] Das Einzige, was in einem solchen Fall funktioniert - deshalb habe ich gesagt, dass wir die Türen schließen müssen ... Die Türen sind leider nicht [...]. “4 Gleichzeitig strömten mehr und mehr Abgeordnete in das Plenum und nahmen ihre Plätze ein.

$\mathrm{Zu}$ diesem Zeitpunkt lässt sich einem Wortaustausch zwischen dem Schriftführer Daniel Sieveke (CDU) und Böth entnehmen, dass beide das Ergebnis eindeutig festgestellt hatten. Sieveke: „Die Reihen von CDU und FDP waren weitaus mehr gefüllt als von SPD und Grünen.“ Böth: „Ja, das ist unstrittig.“ Dieser Dialog ist jedoch nicht protokolliert worden, sondern wurde zufällig vom Videomitschnitt des Landtags festgehalten. ${ }^{5}$ Nach weiterem Zeitverlauf bat Böth zur Beratung des Sitzungsvorstandes, dem die sitzungsleitende Präsidentin sowie die beiden Schriftü̈hrer angehören. ${ }^{6}$ Dieser beschloss während seiner sechsminütigen Beratung eine Wiederholung der Abstimmung (Gegenprobe), da das Ergebnis nicht eindeutig festgestellt worden sei. Dieses Verfahren ist durch die Geschäftsordnung legitimiert und formal korrekt durchgeführt worden. Auf die erste Abstimmung folgte Uneinigkeit im Sitzungsvorstand über das Ergebnis. Daraufhin folgte nach Beratung eine Gegenprobe, deren Ausgang später anerkannt wurde.

Mit Verweis auf die Geschäftsordnung führte Böth anschließend, dreizehn Minuten nach dem ersten Votum, diese Wiederholung durch. Dabei bat sie diejenigen Parlamentarier, nicht an der Abstimmung teilzunehmen, die bei der ersten abwesend waren. Die Hand-

2 Sitzverteilung im Nordrhein-Westfälischen Landtag: CDU 67, SPD 67, Bündnis 90/Die Grünen 23, FDP 13, Linke 11.

3 Vgl. http://real:8080/ramgen/plenum20110519.rm (Abruf am 24. Mai 2011).

4 Gunthild Böth, in: Landtag Nordrhein-Westfalen, Plenarprotokoll 15/35, S. 3422.

5 Vgl. a.a.O (Fn. 3).

6 Neben Daniel Sieveke die zweite Schriftführerin Regina Kopp-Herr (SPD). 
zeichen wurden von den Schriftführern ausgezählt und das Ergebnis von 52 Stimmen für (CDU, FDP) und 57 gegen den Oppositionsantrag (SPD, Grüne, Linke) festgestellt. ${ }^{7}$

Darauf folgte eine Geschäftsordnungsdebatte mit anschließender zweistündiger Sitzungsunterbrechung, in der der Ältestenrat über die Situation beriet. Nach Wiederbeginn der Plenarsitzung verkündete Landtagspräsident Eckhard Uhlenberg (CDU), dass die zweite Abstimmung anerkannt werde. Gleichzeitig kündigte er an, dass der Ältestenrat über mögliche Veränderungen im Abstimmungsvorgang beraten werde.

Die geschilderte Situation im Düsseldorfer Landtag wirft eine Vielzahl parlamentarischer Fragestellungen auf. Davon sollen drei Aspekte nachfolgend behandelt werden: Nach einer kurzen Betrachtung der verfassungsrechtlichen Grundlagen des Abstimmungsprozesses ist erstens zu analysieren, welche Rolle der Sitzungsvorstand, insbesondere der Sitzungspräsident, bei dem Abstimmungsverfahren spielt. Konkreter Gegenstand der Analyse ist sein möglicher Einfluss auf die Abstimmung. Zweitens ist nach den Auswirkungen der Abstimmungsmethoden auf den parlamentarischen Prozess zu fragen. Drittens sind auf dieser Grundlage Reformmöglichkeiten für eine bessere Transparenz bei Abstimmungen zu erörtern.

\section{Verfassungsrechtliche Aspekte parlamentarischer Abstimmungsprozesse}

Zur Stimmabgabe der Abgeordneten in der Plenarsitzung legt die Verfassung des Landes Nordrhein-Westfalen in Artikel 30 Abs. 2 zunächst fest: „Die Abgeordneten stimmen nach ihrer freien, nur durch die Rücksicht auf das Volkswohl bestimmten Überzeugungen; sie sind an Aufträge nicht gebunden." Des Weiteren heißt es bezüglich der Ausübung des Mandats in Artikel 46 Abs. 1: „Abgeordnete dürfen an der Übernahme und Ausübung ihres Mandats nicht gehindert oder hierdurch in ihrem Amt oder Arbeitsverhältnis benachteiligt werden."

Der Düsseldorfer Eklat steht in zwei Aspekten im Widerspruch hierzu. Wenn die sitzungsleitende Landtagspräsidentin dazu auffordert, die Türen des Plenarsaals zu schließen, um die Mehrheitsverhältnisse eindeutig feststellen zu können, werden die Abgeordneten (diejenigen, die noch nicht anwesend waren) gehindert, an der Plenarsitzung und der damit verbundenen Abstimmung teilzunehmen. Die Ausübung ihres Mandats wird eingeschränkt. Zweitens widerspricht die auffordernde Bitte der Präsidentin, dass nur jene Abgeordnete an der Wiederholung (Gegenprobe) teilnehmen mögen, die bereits an der ersten Abstimmung teilgenommen haben, der Landesverfassung. Zum einen stellt dies eine Einschränkung des freien Mandats dar. Zum anderen sind die Parlamentarier nach Art. 30 Abs. 2 an Aufträge nicht gebunden.

\section{Rolle und Einfluss des Sitzungsvorstandes aufZeitpunkt und Durchführung parlamentarischer Abstimmungen}

Vor Beginn einer Plenarsitzung beschließt der Ältestenrat des Parlaments eine Tagesordnung, die Redezeiten der Fraktionen sowie der Regierung. Anschließend wird diese Tagesordnung mit den errechneten Abstimmungszeitpunkten, als Summe der Redezeiten, veröf-

7 Vgl. Landtag Nordrhein-Westfalen, Beschlussprotokoll 15/35. 
fentlicht. Sie bietet somit eine Richtschnur zur Orientierung für Abgeordnete, Journalisten und die Öffentlichkeit. Durch Ausdehnung der Redezeiten oder Verzicht auf Redebeiträge zeigt sich im parlamentarischen Alltag die Flexibilität der Tagesordnungen. ${ }^{8}$

Sind nach einer Parlamentsdebatte keine weiteren Redebeiträge der Parlamentarier erwünscht, so bittet der sitzungsleitende Präsident zur Abstimmung des Tagesordnungspunktes. Geleitet und durchgeführt wird die Abstimmung durch den Sitzungsvorstand. Dieser wird laut Geschäftsordnung des Landtags von Nordrhein-Westfalen durch den amtierenden Präsidenten und die amtierenden Schriftführer gebildet ( $\$ 8$ Abs. 1 GO). Aufgabe der Schriftführer ist es unter anderem, „die Stimmen zu sammeln und zu zählen“ ( $\$ 8$ Abs. 2 GO). Einstimmig stellt der Sitzungsvorstand nach einer Abstimmung das Ergebnis fest, das durch den amtierenden Präsidenten verkündet wird ( $\$ 45$ Abs. 1 GO). Ist der Sitzungsvorstand sich über das Ergebnis der Abstimmung nicht einig, wird diese wiederholt (Gegenprobe). Sollten auch danach die Mitglieder des Sitzungsvorstandes das Ergebnis nicht einstimmig feststellen, werden die Stimmen nach dem so genannten Hammelsprungverfahren gezählt ( $\$ 42$ Abs. 5 GO).

Die Geschäftsordnung regelt also den Prozess der Abstimmung und die Feststellung des Ergebnisses. Die Parlamentarier geben per Handzeichen ihr Votum ab, die beiden Schriftführer erheben das Ergebnis. Problematisch ist jedoch der Zeitpunkt des Votums. Bittet der Präsident um Handzeichen, so beginnen die Schriftführer mit der Auszählung. Bei 181 Abgeordneten im Düsseldorfer Landtag dauert dieser Vorgang somit einige Augenblicke an. Während dieses Zeitraums ist eine Veränderung der Mehrheitsverhältnisse durch Hereinkommen weiterer Parlamentarier in den Sitzungssaal denkbar. Es entsteht eine unübersichtliche Situation für das Auszählen der Stimmen, so dass zu fragen ist, ob die verspätet eingetretenen Parlamentarier noch an der Stimmabgabe teilnehmen dürfen.

Bei einer geheimen Abstimmung ist der Abstimmungszeitraum eindeutig. Der Präsident fordert die Abgeordneten auf, ihre Stimme abzugeben und eröffnet die Abstimmung. Abschließend fragt er, ob noch weitere Stimmen abzugeben sind. Ist dies nicht der Fall, wird die Abstimmung geschlossen. Es existiert ein definierter Abstimmungszeitraum. Bei der Methode der namentlichen Abstimmung bemisst die Geschäftsordnung einen ähnlich definierten Abstimmungszeitraum. Die Parlamentarier werden namentlich um ihr Votum gebeten. Erfolgt dieses nicht, da der Abgeordnete nicht anwesend ist, wird sein Name wiederholt. Unterlässt er weiterhin, seine Stimme abzugeben, wird der nächste aufgerufen. Abschließend erfolgt auch hier die Frage, ob jemand seine Stimme noch nicht abgegeben hat. Ein bei seinem Namensaufruf noch nicht anwesender Abgeordneter kann sein Votum an dieser Stelle noch nachholen. Die Abstimmung wird nun geschlossen, und es wird ausgezählt. Der Abstimmungszeitraum ist auch hier eindeutig definiert. Werden die Parlamentarier aufgefordert, ihre Zustimmung, Ablehnung oder Enthaltung durch Handheben kenntlich zu machen, umfasst der Abstimmungszeitraum jeweils den Zeitpunkt von der Aufforderung zur Abstimmung bis zum Abschluss des Auszählens. Bei dieser Methode ist es jedoch nicht gängig, dass der Präsident nach Stimmabgabe ins Plenum fragt, ob jeder seine Stimme abgegeben hat.

8 Vgl. Susanne Linn / Frank Sobolewski, So arbeitet der Deutsche Bundestag, Rheinreitbach 2010, S. $22 \mathrm{f}$. 
Wie oben beschrieben findet laut Geschäftsordnung bei nicht einstimmiger Feststellung des Abstimmungsergebnisses durch den Sitzungsvorstand eine Wiederholung der Abstimmung statt. Das Verhalten der sitzungsleitenden Präsidentin im Düsseldorfer Landtag wirft dabei die Frage auf, ob die Wiederholung einer Abstimmung eine Rekonstruktion des Votums bedeutet und damit nur die Teilnehmer des ersten erneut abstimmen dürfen. Achterberg schreibt dazu: „Die Möglichkeit der Abstimmungswiederholung wird im Übrigen mit dem gewohnheitsrechtlich, mitunter verfassungsrechtlich geltenden Prinzip der Unverrückbarkeit der Parlamentsbeschlüsse in Zusammenhang gebracht. Überzeugend ist dies indessen nicht, denn dieses Prinzip behielte auch dann Gewicht, wenn man beispielsweise die Wiederholung der Abstimmung wegen Irrtums zuließe; es bedeutet dann eingeschränkt, dass nur eine fehlerfrei vorgenommene Abstimmung nicht wiederholt werden kann. [...] Bei der Abstimmungswiederholung wegen Irrtums ist vorab festzustellen, dass die bürgerlichrechtlichen Vorschriften über den Irrtum hier nicht anwendbar sind [...]. Aber darüber hinaus wird die Wiederholung der Abstimmung für zulässig erachtet, wenn es sich um einen Irrtum in der Sache oder über die Auswirkung der Abstimmung, nicht dagegen nur um einen solchen im Verhalten oder im Mittel handelt. " 9 Und weiter, direkt einschlägig für die geschilderte Situation im Düsseldorfer Landtag: „Auch die Anzweifelung des Abstimmungsergebnisses kann nicht zur Wiederholung der Abstimmung führen; dasselbe gilt für die Unterstellung von Unregelmäßigkeiten im Abstimmungsverfahren. [...] Unerheblich für die Zulässigkeit der Abstimmungswiederholung ist es, ob der Irrtum durch die Fragestellung verursacht wurde und/oder ein anderes Abstimmungsergebnis zu erwarten ist, da hierüber präzise Prognosen nicht immer möglich sind." Grundsätzlich ist zudem festzustellen, dass die Wiederholung einer Abstimmung laut Geschäftsordnung bedeutet, dass ein neuer Wahlgang, unabhängig vom ersten Votum, vollzogen wird ( $\$ 42 \mathrm{Abs.} 5 \mathrm{GO}$ ). Damit entstehen bei der Wiederholung eine neue Teilnehmerschaft sowie ein neuer Abstimmungszeitraum.

Der beschriebene Abstimmungsprozess aus dem Düsseldorfer Landtag lässt eine weitere Frage bezüglich des Einflusses des Sitzungsvorstandes auf das Abstimmungsergebnis entstehen. Die Geschäftsordnung sieht vor, dass der Sitzungsvorstand, zusammengesetzt aus drei Vertretern, das Abstimmungsergebnis einstimmig feststellen muss. Dabei ist anzumerken, dass die beiden Schriftführer jeweils einer Regierungs- und einer Oppositionsfraktion angehören müssen. Ein politisch einseitig besetzter Sitzungsvorstand soll damit ausgeschlossen werden. ${ }^{10}$ An dieser Stelle ist eine Besonderheit einer Minderheitsregierung im parlamentarischen Regierungssystem festzustellen: Da eine Minderheitsregierung die Unterstützung von mindestens einer der Oppositionsfraktionen benötigt, ist ein politisch einseitig besetzter Sitzungsvorstand nicht auszuschließen. Stimmt beispielsweise die FDP mit den Regierungsfraktionen für einen Gesetzentwurf, so ist es möglich, dass zu diesem Zeitpunkt der Sitzungsvorstand durch Vertreter von FDP, SPD und Grünen gebildet wird. Die bei dieser Abstimmung tatsächlich als Opposition fungierenden Fraktionen haben keinerlei Möglichkeit, bei Unstimmigkeiten im Abstimmungsverfahren durch den Sitzungsvorstand Einfluss zu nehmen. Somit sind aufgrund der wechselnden Mehrheiten bei einer Minderheitsregierung die Rechte der Opposition im Sitzungsvorstand prinzipiell eingeschränkt.

9 Norbert Achterberg, Parlamentsrecht, Tübingen 1984, S. 649 f.

10 Vgl. Heinrich G. Ritzel / Joseph Bücker / Hermann J. Schreiner, Handbuch für die parlamentarische Praxis, Berlin 2008, $\$ 51$. 
Grundsätzlich ist festzuhalten, dass Abstimmungsergebnisse nur dann aktiv durch den Sitzungsvorstand verändert werden können, wenn alle drei Mitglieder dies einstimmig feststellen. Es bedarf somit einer Handlung aller drei Mitglieder des Sitzungsvorstandes. Es besteht jedoch auch die Möglichkeit zur passiven Beeinflussung des Abstimmungsergebnisses durch zeitliche Aufschiebung seiner Feststellung. Legt ein Vorstandsmitglied sein Veto ein - dies ist ohne Begründung möglich -, muss die Abstimmung wiederholt werden. ${ }^{11}$ „Über die Feststellung des Präsidenten, dass der Sitzungsvorstand über das Abstimmungsergebnis nicht einig ist, kann nicht diskutiert werden. Sie ist auch nicht anfechtbar." 12 Jedes Mitglied des Sitzungsvorstandes besitzt somit die Möglichkeit, einen Aufschub herbeizuführen, wie er im Düsseldorfer Landtag dreizehn Minuten angedauert hat. Auch wenn es sich nur um wenige Minuten handelt, kann sich das Mehrheitsverhältnis im Plenum erheblich verändern. Mit der Verschiebung des Abstimmungszeitpunktes besitzt der Sitzungsvorstand also im parlamentarischen Alltag eine passive Einflussmöglichkeit auf den Abstimmungsvorgang. Diese kann sich im Übrigen auch durch Unsicherheit über den protokollarischen Ablauf, ergeben. Der Einfluss des Präsidiums kann somit in zweierlei Form ausgeübt werden: Zum einen, wie dem Beispiel zu entnehmen ist, wird durch Verzögern der Abstimmung den Fraktionen die Möglichkeit eingeräumt, vollzählig teilzunehmen und somit die durch die Wahl erzeugten demokratischen Mehrheitsverhältnisse zum Tragen zu bringen. Zum anderen besteht aber auch die Einflussmöglichkeit, durch eine besonders schnelle Abwicklung des Abstimmungsvorganges, kurzfristig entstandene Mehrheitsverhältnisse zu nutzen. Beide Varianten stellen einen passiven Spielraum zur Beeinflussung des Abstimmungsvorganges dar.

\section{Abstimmungsmethoden im parlamentarischen Prozess}

Grundsätzlich stehen im Düsseldorfer Landtag wie auch im Deutschen Bundestag unterschiedliche Varianten der Abstimmung zur Verfügung. Diese lassen sich systematisch nach der Form und der Offenkundigkeit des Abstimmungsverhaltens untergliedern. Der Form nach gibt es die mündliche Abstimmung (durch Namensruf und Erklärungsantwort), durch Zeichen - insbesondere durch Handzeichen oder Aufstehen und Sitzenbleiben - sowie die schriftliche Abstimmung. Der Offenkundigkeit nach lassen sich offene und geheime Abstimmungen unterscheiden. Mündliche Abstimmungen und Abstimmungen durch Zeichen sind ihrer Natur nach offen, Abstimmungen mit „verdeckten Stimmzetteln“ demgegenüber stets geheim, während umgekehrt geheime Abstimmungen nicht nur in dieser Form durchführbar sind. ${ }^{13}$ In der Regel erfolgt die Abstimmung per Handzeichen. ${ }^{14}$

Das Handheben als Abstimmungszeichen, in Nordrhein-Westfalen variabel durch Aufstehen zu ersetzen, ist eine eindeutige Methode, die durch die Schriftführer wie auch durch die Öffentlichkeit erkannt und zugeordnet werden kann. Dabei erfolgt die Auswertung mittels Auszählen durch die Schriftführer.

11 Vgl. Susanne Linn / Frank Sobolewski, a.a.O. (Fn. 8), S. 18 f.

12 Hans Trossmann, Parlamentsrecht des Deutschen Bundestages, München 1977, S. 390.

13 Vgl. Norbert Achterberg, a.a.O. (Fn. 9), S. 641.

14 Zudem existiert in den meisten Parlamenten die Abstimmungsmethode der geheimen Stimmabgabe, die an dieser Stelle vernachlässigt wird. 
Nach der Bitte um Handzeichen ist es Aufgabe der Schriftführer die Stimmen zu zählen. Dabei kann zum einen bei der Vielzahl von Mandatsträgern (gegenwärtig 181 im Düsseldorfer Landtag) ein Abgeordneter übersehen werden, wodurch das Ergebnis verfälscht wird. Dies kann absichtlich wie unabsichtlich geschehen. Jedoch sollte durch die zweifache Auszählung, die durch die beiden Schriftführer durchgeführt wird, die Korrektheit des Ergebnisses gewährleistet sein.

Zum zweiten haben auch Mitarbeiter der Fraktionen Zugang zum Plenarsaal, so dass sie den Platz eines Abgeordneten einnehmen können. Bei der Vielzahl von Parlamentariern kann nicht leicht festgestellt werden, ob Abgeordnete auf ihren Plätzen sitzen oder es sich um Mitarbeiter der Fraktionen handelt. ${ }^{15}$

Drittens existiert eine weitere mögliche Fehlerquelle durch die Sitzordnung des Ministerpräsidenten und der Minister. Die Mitglieder der Landesregierung mit Abgeordnetenmandat haben zwei Stühle im Plenarsaal, einen auf der Regierungsbank, einen in ihrer jeweiligen Fraktion. Trotz Anwesenheit der jeweiligen Person existiert somit gleichzeitig ein leerer Stuhl. Diese Lücke im Plenarsaal bietet eine Fehlerquelle beim Auszählen durch die Schriftführer. Im Bundestag ist diese „Doppelbestuhlung“ eindeutig geregelt. „Es entspricht aber langer parlamentarischer Tradition, dass die Stimmabgabe der Regierungsmitglieder mit Abgeordnetenmandat von den Abgeordnetenplätzen und nicht von der Regierungsbank aus erfolgt. Der Grundgedanke, dass von der Regierungsbank aus keine Abgeordnetenrechte ausgeübt werden sollen, bleibt damit einheitlich gewahrt. [...] Bei unübersichtlichen oder knappen Mehrheitsverhältnissen müssen sich Regierungsmitglieder mit Abgeordnetenmandat deshalb zu den Abgeordnetenplätzen begeben, um von dort aus ihre Stimme abzugeben. [...] In der 13. WP ist ein Vorschlag der Bundesregierung, Mitgliedern der Bundesregierung mit Abgeordnetenmandat künftig die Möglichkeit der Stimmabgabe von der Regierungsbank aus einzuräumen, vom Präsidium und vom Ältestenrat abgelehnt worden. "16

Viertens erfordert das Auszählen durch die Schriftführer erheblichen zeitlichen Aufwand. Bei 181 Abgeordneten im Düsseldorfer Landtag bedurfte das Auszählen der oben beschriebenen Wiederholung der Abstimmung rund drei Minuten. Während dieser Zeit konnten weitere Parlamentarier den Sitzungssaal betreten und zusätzlich an der Abstimmung teilnehmen.

Zusammenfassend ist festzuhalten, dass Sitzungsvorstand und Öffentlichkeit durch die Methode der Abstimmung die Möglichkeit haben müssen, das Verhalten jedes einzelnen Parlamentariers und damit das Ergebnis eindeutig zu erkennen. Ein Perspektivwechsel über den Nordrhein-Westfälischen Landtag hinaus verdeutlicht, dass weitere Verfahren existieren, um parlamentarische Abstimmungen durchzuführen. Diese Regelungen finden sich in den Geschäftsordnungen oder vergleichbaren Regelwerken der Parlamente. Die Übersicht in Tabelle 1 verdeutlicht die Bandbreite in sieben Ländern.

Interessanterweise war auch im Deutschen Bundestag die Abschaffung des Hammelsprungs bereits beschlossen. Ab Ostern 1970 stimmten die Parlamentarier mittels der Computer-Anlage AEG 60-10-Digitalrechner ab: „Die Idee der gesteuerten Abstimmung, um die sich bis Ostern 20 Elektroniker fieberhaft kümmern, damit den Abgeordneten das ständige Handheben und der Hammelsprung erspart bleiben, ist schon 20 Jahre alt. Der

15 Vgl. Michael Püschner, Der Fraktionsreferent - ein politischer Akteur?, in: APuZ, 38/2009, S. 33 -38 .

16 Heinrich G. Ritzel / Joseph Bücker / Hermann J. Schreiner, a.a.O (Fn. 10), \$48. 


\begin{tabular}{|c|c|c|c|}
\hline Land & Parlament & Methoden & Besonderheiten \\
\hline Deutschland & Bundestag & $\begin{array}{l}\text { Handzeichen, Aufstehen, } \\
\text { namentliche Abstim- } \\
\text { mung, Hammelsprung }\end{array}$ & $\begin{array}{l}\text { In der abschließenden Lesung eines } \\
\text { Gesetzentwurfes muss per Aufstehen, } \\
\text { nicht per Handzeichen abgestimmt } \\
\text { werden. }\end{array}$ \\
\hline Deutschland & $\begin{array}{l}\text { Landtag von } \\
\text { Nordrhein- } \\
\text { Westfalen } \\
\end{array}$ & $\begin{array}{l}\text { Handzeichen, Aufstehen, } \\
\text { Namensaufruf, Hammel- } \\
\text { sprung }\end{array}$ & ( \\
\hline USA & Senat & $\begin{array}{l}\text { Zuruf im Chor, } \\
\text { Namensaufruf }\end{array}$ & $\begin{array}{l}\text { Auf Frage des Sitzungspräsidenten } \\
\text { antworten die Senatoren im Chor } \\
\text { nacheinander mit „Aye“ (Zustim- } \\
\text { mung) oder „Nay“ (Ablehnung). } \\
\text { Der Sitzungspräsident verkündet } \\
\text { danach das Ergebnis nach seinem } \\
\text { akustischen Eindruck. } \\
\text { Auf Wunsch oder Unklarheit erfolgt } \\
\text { eine namentliche Abstimmung. }\end{array}$ \\
\hline USA & $\begin{array}{l}\text { Repräsentanten- } \\
\text { haus }\end{array}$ & $\begin{array}{l}\text { Zuruf im Chor, } \\
\text { Namensaufruf, elektro- } \\
\text { nische Wahlkarte }\end{array}$ & $\begin{array}{l}\text { Siehe US-Senat. } \\
\text { Abgeordnete besitzen zudem eine } \\
\text { elektronische Wahlkarte, mit der sie } \\
\text { an einer von } 44 \text { Wahlurnen ihre } \\
\text { Stimme abgeben können. }\end{array}$ \\
\hline $\begin{array}{l}\text { Groß- } \\
\text { britannien }\end{array}$ & $\begin{array}{l}\text { House of } \\
\text { Commons }\end{array}$ & $\begin{array}{l}\text { Zuruf im Chor, } \\
\text { Hammelsprung }\end{array}$ & $\begin{array}{l}\text { Siehe US-Senat. Ist der Zuruf im } \\
\text { Chor nicht eindeutig, wird per } \\
\text { Hammelsprung abgestimmt. }\end{array}$ \\
\hline Russland & Duma & $\begin{array}{l}\text { Abstimmung per } \\
\text { Knopfdruck }\end{array}$ & $\begin{array}{l}\text { An jedem Abgeordnetenplatz sind } \\
\text { drei Knöpfe (Ja, Nein, Enthaltung) } \\
\text { montiert. Der Abstimmungszeitraum } \\
\text { beträgt } 20 \text { Sekunden. }\end{array}$ \\
\hline Japan & Kokkai & $\begin{array}{l}\text { Abstimmung auf Stimm- } \\
\text { zetteln, Aufstehen }\end{array}$ & $\begin{array}{l}\text { Stimmabgabe mittels zwei Stimmzet- } \\
\text { teln, zum einen die getroffene Wahl } \\
\text { (weißer Zettel), zum anderen der } \\
\text { eigene Name (grüner Zettel). }\end{array}$ \\
\hline Schweiz & Nationalrat & $\begin{array}{l}\text { Elektronisches Verfahren, } \\
\text { Aufstehen, Namensaufruf }\end{array}$ & $\begin{array}{l}\text { Das elektronische Abstimmungs- } \\
\text { system zählt und speichert die } \\
\text { abgegebenen Stimmen. Das Stimm- } \\
\text { verhalten der Ratsmitglieder und das } \\
\text { Resultat werden auf Anzeigetafeln an- } \\
\text { gezeigt. Bei geheimer Beratung oder } \\
\text { falls die elektronische Abstimmungs- } \\
\text { anlage defekt ist, erfolgt die Stimm- } \\
\text { abgabe durch Aufstehen oder durch } \\
\text { Namensaufruf. }\end{array}$ \\
\hline Frankreich & $\begin{array}{l}\text { National- } \\
\text { versammlung }\end{array}$ & $\begin{array}{l}\text { Handzeichen, Aufstehen, } \\
\text { namentliche Abstimmung } \\
\text { (i.d.R. elektronisches Ver- } \\
\text { fahren, ansonsten Stimm- } \\
\text { zettel) }\end{array}$ & $\begin{array}{l}\text { Das elektronische Verfahren bei der } \\
\text { namentlichen Abstimmung umfasst } \\
\text { lediglich die Stimmerfassung. Eine } \\
\text { Wahlkarte muss weiterhin in eine } \\
\text { Wahlurne gegeben werden. }\end{array}$ \\
\hline
\end{tabular}


SPD-Abgeordnete Karl Mommer besuchte 1950 amerikanische Staatsparlamente, sah diese „Wahlmethode“ und trug sie dem Organisationsausschuss des Bundestages vor. [...] Akut wurde die Idee erst wieder 19 Jahre später [...]. So holte Präsident von Hassel die Pläne wieder aus der Schublade [...]. Gegenwärtig sind Elektriker und Techniker dabei, die Pulte und Sessel zu „frisieren“. Auf den Schreibtischen der Abgeordneten werden Knopfdruckanlagen installiert, die zwischen „Ja“, „Nein“, „Enthaltung“ und „Storno“ unterscheiden und für Debatten die Tasten „Wortmeldung“ und „Zwischenfrage“ bereithalten. “17 Die Verwendung der elektronischen Abstimmungsanlage in Bonn war jedoch nicht von langer Dauer: „Nach mehreren missglückten Versuchen, mit der 1970 installierten Abstimmungsanlage Abstimmungen durchzuführen, unternahm der Ältestenrat im Oktober 1973 einen erneuten Anlauf. [...] Nach den Erfahrungen in dieser Sitzung ist die Anlage nie wieder betätigt worden. Sie wurde stattdessen einige Zeit später vollständig abgebaut. "18 Der Bundestag kehrte zu den gewohnten Methoden des Handhebens, Aufstehens oder des Hammelsprungs zurück.

\section{Reformmöglichkeiten des Abstimmungsprozesses}

Wie aus obiger Tabelle ersichtlich besitzen diverse Parlamente elektronische Abstimmungsanlagen beziehungsweise unterstützende technische Hilfsmittel zur Feststellung der Abstimmungsergebnisse. Die Einführung solcher Geräte erscheint als die nächstliegende Reformmethode. Mit diesen könnte das Abstimmungsverhalten im Plenarsaal zum entsprechenden Zeitpunkt dokumentiert und dem Sitzungsvorstand keinerlei Beeinflussungsspielraum überlassen werden. Die Technik läuft im Hintergrund und kann in unklaren Abstimmungssituationen vom Sitzungsvorstand eingesehen werden. Dieser behält die Kompetenz der einstimmigen Ergebnisfeststellung. Zudem wird mit dieser Methode ein eindeutiger Zeitraum für die Abstimmung festgelegt. Die Parlamentarier, die nicht innerhalb dieses Zeitraums abstimmen, nehmen nicht an dem Votum teil.

Zweifelsohne können als positive Effekte solcher technischer Lösungen Zeitersparnis, Genauigkeit und Transparenz verbucht werden. Gegen sie sprechen aber eine Reihe von Schwierigkeiten, die sich aus den Erfahrungen der Parlamente in anderen Ländern ablesen lassen. So ist es beispielsweise gar nicht einfach sicherzustellen, dass Parlamentarier bei ihrer elektronischen Stimmabgabe persönlich im Plenarsaal anwesend sind und ihre Stimme eigenständig abgeben oder dass nicht ein Abgeordneter stellvertretend für viele votiert etc. Daher können elektronische Hilfsmittel bei der Feststellung von Abstimmungsergebnissen Unterstützung leisten, solange sie den parlamentarischen Prozess nicht beeinflussen. Ihr Einsatz sollte aber jederzeit durch klassische Abstimmungsmethoden ersetzt werden können, damit keine Abhängigkeit entsteht.

Um eine angemessene Lösung zu unterbreiten, damit Fälle wie der aus dem NordrheinWestfälischen Landtag geschilderte vermieden werden können, bedarf es zunächst der

17 Bernd Leyendecker, Ab Ostern stimmt das Parlament mit Knopfdruck ab, in: Das Parlament, 20. Jg. (1970), H. 7, S. 11.

18 Peter Schindler, Datenhandbuch zur Geschichte des Deutschen Bundestages 1949 bis 1999, Baden-Baden 1999, S. 1729 f. 
Vergegenwärtigung der komplexen Alltagswirklichkeit bei parlamentarischen Abstimmungen, die in der wissenschaftlichen Literatur selten erfasst ist.

Am Beispiel des Düsseldorfer Landtags: In der Regel beginnen Plenarsitzungen um 10:00 Uhr. Eine Sitzungswoche umfasst zwei bis drei Plenartage, die je nach Umfang der Tagesordnung bis in den späten Abend andauern können. Die durchschnittliche Sitzungsdauer in der abgelaufenen 14. Wahlperiode umfasste 9:06 Stunden. Damit endete die durchschnittliche Plenarsitzung um 19:06 Uhr. ${ }^{19}$

Während eines Plenartages kommt es rund alle 60 bis 90 Minuten zur Abstimmung im Plenarsaal. Dabei kann es sich um eine namentliche Abstimmung über das Haushaltsgesetz oder lediglich um eine Ausschussüberweisung handeln. In der Regel nehmen nur jene Abgeordnete an den Sitzungen teil, die für ihre Fraktion Experten auf dem jeweils anstehenden Gebiet sind. Bei der Debatte zur Schulpolitik ist ein Umweltausschussmitglied normalerweise nicht anwesend. Anderes gilt für Generaldebatten oder Regierungserklärungen. Aus dieser Praxis ergibt sich, dass die meisten Parlamentarier erst zu den Abstimmungen den Plenarsaal betreten. Per Kurznachricht, Anruf oder Lautsprecherdurchsage erhalten sie die Information über eine bevorstehende Abstimmung und die Bitte der parlamentarischen Geschäftsführung zur Teilnahme. In den Zeit zwischen den Abstimmungen finden Konferenzen, Arbeitsgespräche oder auch Bürotätigkeiten statt. Die langen Plenartage und Abstimmungen im Rhythmus von 60 bis 90 Minuten führen zu einem nur mäßig besuchten Plenarsaal, und obwohl dies gute Gründe hat, trägt dies zu einem negativen Parlamentsund Abgeordnetenbild in der Öffentlichkeit bei und weckt Zweifel an der demokratischen Legitimität der Abstimmungen. Hier ist eine realitätsgerechte und damit verbesserte öffentliche Wahrnehmung von Parlamenten und ihren Mitgliedern dringend vonnöten.

Grundsätzliche Voraussetzung für demokratischen Parlamentarismus ist die Nachvollziehbarkeit und Transparenz der Entscheidungen. So kann man beispielsweise in Nordrhein-Westfalen mit wenig Aufwand die Plenarreden eines einzelnen Abgeordneten bekommen, jedoch ist es nahezu unmöglich, sein Verhalten in der darauffolgenden Abstimmung zu erfahren, denn bei nicht-namentlichen Abstimmungen - die Normalität in deutschen Parlamenten - wird lediglich festgehalten, wie die Fraktionen votieren. Äußert ein Abgeordneter sich nicht durch eine gesonderte persönliche Erklärung, die er zu Protokoll geben kann, wird sein Votum automatisch dem seiner Fraktion zugerechnet. Nur bei namentlichen Abstimmungen, die von mindestens einer Fraktion oder einem Viertel der Anwesenden beantragt werden können ( $\$ 43$ Abs. 1 GO LT-NRW), ist das Votum aller Parlamentarier protokollarisch dokumentiert. ${ }^{20}$

Diese Praxis ist Ergebnis eines zeitsparenden Prozesses im arbeitsteiligen Parlamentarismus. Die Fachpolitiker erarbeiten die inhaltlichen Positionen, denen sich (nach einem entsprechenden Willensbildungsprozess in der Fraktion) ihre Fraktionskollegen mit jeweils anderen Arbeitsschwerpunkten anschließen. Suzanne S. Schüttemeyer fasst diesbezüglich

19 Vgl. Landtag Nordrhein-Westfalen, Plenarprotokoll 14/1 bis 14/149.

20 So auch im Deutschen Bundestag: „Bei den namentlichen Abstimmungen wird die Entscheidung des einzelnen MdB für die Öffentlichkeit feststellbar und nachprüfbar, weil die Voten der MdB in einer Liste im Stenographischen Bericht festgehalten werden. "Siehe Heinrich G. Ritzel / Joseph Bücker / Hermann J. Schreiner, a.a.O. (Fn. 10), \$52. Heutzutage erfolgt die Unterrichtung der Öffentlichkeit sofort nach Feststellung des amtlichen Ergebnisses der Abstimmung über das Internet und im Übrigen auch durch die Fraktionen. 
den parlamentarischen Fokus auf Fraktionen wie folgt zusammen: „Aus der Perspektive der Parlamente sind Fraktionen ihre unverzichtbaren Binnenorganisationen, um politische Entscheidungen vorzustrukturieren, den parlamentarischen Prozess zu steuern und - im parlamentarischen Regierungssystem besonders wichtig - durch Geschlossenheit für stabile Mehrheiten zu sorgen, so dass eine Regierung kalkulierbar handlungsfähig ist, die Opposition konkret alternativfähig gemacht wird. "21 Demnach sind Fraktionen nicht nur Organisationseinheiten, sondern entscheidender Faktor in der parlamentarischen Meinungs- und Mehrheitsbildung.

Unter dieser Perspektive ist es entbehrlich festzuhalten, ob ein Abgeordneter an der Abstimmung teilgenommen hat oder (ohne Abgabe einer persönlichen Erklärung) mit oder entgegen seiner Fraktion gestimmt hat. Da folglich nur das Votum der Fraktionen als Ganze erfasst wird ${ }^{22}$, wirkt die Mehrzahl der Abstimmungen für die Öffentlichkeit wenig transparent und nachvollziehbar. Dies widerspricht der oben genannten Anforderung an eine parlamentarischen Demokratie.

Angesichts der Logik des Parlamentarismus sollte hieraus die Konsequenz gezogen werden, immer - wenn es keinen Antrag auf namentliche Abstimmung gibt - die nominellen Fraktionsstärken bei Abstimmungen zu zählen (bei Bedarf unter Abzug der Abgeordneten, die persönliche Erklärungen abgegeben haben), unabhängig davon, wie viele Parlamentarier an- oder abwesend sind. Damit würden die demokratisch legitimierten Sitzverhältnisse des Parlaments widergespiegelt. Vor allem aber entstünden auf diese Weise keine Zufallsmehrheiten für allgemeinverbindliche Beschlüsse. Auch wenn der Opposition damit ein Stück Parlamentshandwerk genommen würde, um die Regierung unter Druck zu setzen: Die Wahrung der demokratisch legitimierten Mehrheitsverhältnisse ist höher zu werten. Schließlich ist nicht zu rechtfertigen, dass ein organisatorisches Fehlverhalten von Abgeordneten der Regierungsfraktionen zu einer Veränderung der vom Wähler gewollten Mehrheitsverhältnisse führt. Das individuelle Stimmrecht der Parlamentarier ist davon unberührt. Will ein Abgeordneter sich das Stimmverhalten seiner Fraktion nicht zurechnen lassen, kann er sein persönliches Abstimmungsverhalten durch eine Erklärung zur Abstimmung, die auch schriftlich erfolgen kann, offiziell im Protokoll dokumentieren lassen.

Unter dieser Voraussetzung bietet eine Zählung nach nomineller Fraktionsstärke parlamentstheoretische und -praktische Vorteile. Sie gibt der Öffentlichkeit ein realitätsgerechtes Bild der parlamentarischen Repräsentation, denn faktisch stehen die gesamten Fraktionen hinter dem jeweiligen Abstimmungsverhalten im Plenum, das lediglich Schlusspunkt eines umfassenden Willensbildungsprozesses ist. Und sie garantiert, dass die demokratisch legitimierten Mehrheitsverhältnisse zum Tragen kommen. Ein Abstimmungseklat wie im Düsseldorfer Landtag würde unwahrscheinlich.

21 Suzanne S. Schüttemeyer, Fraktionen und ihre Parteien in der Bundesrepublik Deutschland, Opladen 1999, S. 39.

22 Könen verweist diesbezüglich auf die Geschäftsordnungen der Parlamente und die fehlende Verpflichtung der Landtagspräsidenten, „dissentierendes Abstimmungsverhalten verbal festzuhalten“. In keinem der vier von ihr untersuchten Landtage (Brandenburg, Nordrhein-Westfalen, Bayern und Sachsen) existiert eine solche Verpflichtung; Susanne Könen, Wo sind die Rebellen hin? Dissentierendes Abstimmungsverhalten in ost- und westdeutschen Landtagen, Wiesbaden 2009, S. 133. 